\title{
Hardware-Efficient Architecture Design of Wavelet-Based Adaptive Visible Watermarking
}

\author{
Yu-Cheng Fan, Lan-Da Van, Chun-Ming Huang, and Hen-Wai Tsao
}

\begin{abstract}
This paper proposes a hardware-efficient architecture design of wavelet-based adaptive visible watermarking. Our method adopts discrete wavelet transform (DWT) to embed visible watermarking. We perform this algorithm using hardware-efficient architectures that combine operational reduction and resource sharing techniques. Our method provides high quality visible watermarking image and small hardware cost. The experimental results have proven that our method is indeed effective'.
\end{abstract}

Index Terms - discrete wavelet transform, resource sharing, visible watermarking, Weber-Fechner law.

\section{INTRODUCTION}

In this paper, we propose hardware-efficient architecture design of wavelet-based adaptive visible watermarking (Fig. 1). Visible watermarking schemes are important intellectual property right protection mechanisms for digital images and videos that have to be released for certain purposes but illegal reproductions of them are prohibited [1][2]. Visible watermarking techniques protect digital contents in a more active manner, which is quite different from the invisible watermarking techniques [1-5].

Several software based visible watermarking schemes have been presented in the literature; however, only a few hardware schemes have been proposed. In 2004, Mohanty and Ranganathan proposed spatial domain visible watermarking architecture [6]. In 2005, Mohanty presented another watermarking chip that has both visible and invisible discrete cosine transform (DCT) domain watermarking functionalities [7].

Block based DCT domain visible watermarking changes the scaling factors block by block and may result in visual

\footnotetext{
'This work was supported by the, National Chip Implementation Center (CIC), National Applied Research Laboratories.

Yu-Cheng Fan is with the Department of Electrical Eng. and Graduate Institute of Electronics Eng., National Taiwan University, Taipei, Taiwan, R.O.C. (e-mail: d9921004@ee.ntu.edu.tw).

Lan-Da Van is with the National Chip Implementation Center (CIC), National Applied Research Laboratories, Science-Based Industrial Park, Hsinchu 300, Taiwan, R.O.C.

Chun-Ming Huang is with the National Chip Implementation Center (CIC), National Applied Research Laboratories, Science-Based Industrial Park, Hsinchu 300, Taiwan, R.O.C.

Hen-Wai Tsao is with the Department of Electrical Eng. and Graduate Institute of Electronics Eng., National Taiwan University, Taipei, Taiwan, R.O.C.
}

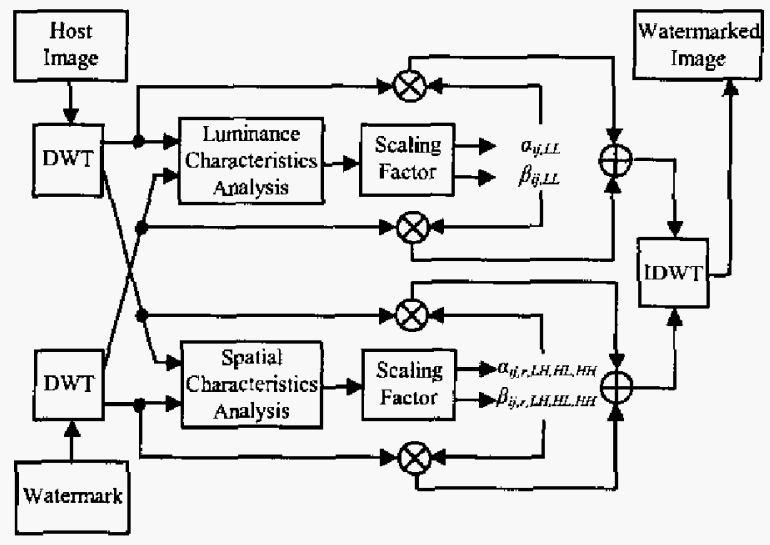

Fig. 1. Block diagram of wavelet-based adaptive visible watermarking.

discontinuity [8]. Therefore, Hu [8] proposed a waveletbased visible watermarking to solve this problem. Waveletbased visible watermarking is applied to JPEG-2000 image and many consumer electronics product. These techniques become more and more important.

However, wavelet-based visible watermarking chip has not been performed until now. In order to develop the efficient hardware architecture of wavelet-based adaptive visible watermarking, we adopt operational reduction and resourcesharing techniques to perform it according to Hu's algorithm [8].

\section{Human Visual System}

In order to design well visible watermarking, we shall concern ourselves with psychophysies, i.e., the response of observers to visual stimulus, the response from physiology, or the structure of the visual apparatus. The response of the eye to changes in the intensity of illumination is nonlinear. Consider a background of intensity $I$ surrounds a patch of light of intensity $I+\Delta I$ (Fig. 2). The just noticeable difference (JND) $\Delta I$ is to be determined as a function of $I$. The ratio $\Delta I / I$, called the Weber fraction (Weber-Fechner law), is nearly constant at a value of about 0.02 [9-11]. However, this result does not hold at very low or very high intensities, as illustrated by Fig. 2. When a patch is at very low or very high intensities, the ratio $\Delta I / I$ appears larger value.

According to Weber-Fechner law, we can regard $I+\Delta I$ as 


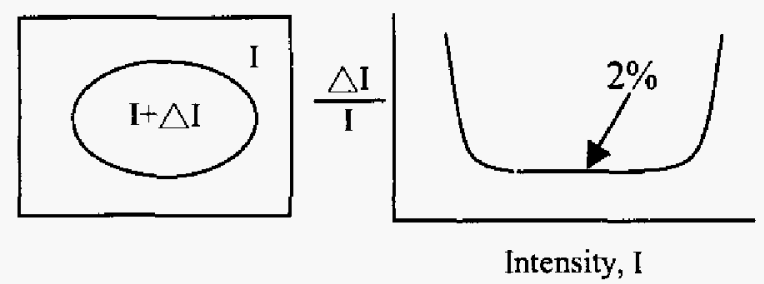

Fig. 2. Weber-Fechner law.

a patch of watermarked image and $I$ as an original image. When the ratio $\Delta l / I$ is greater than Weber fraction, the watermark become visible. The ratio $\Delta I / I$ increases more, the visible watermark becomes more conspicuous.

\section{IIJ. WaVELET-BASEd AdAPTIVE VISIBLE WATERMARKING}

First of all, host image and watermark is transferred by discrete wavelet transform (DWT) (Fig. 1). Then, luminance characteristics are analyzed according to the image structure of wavelet decomposition. The low frequency sub-band image and the original image have similar histogram characteristics. Luminance affects the coefficients in the low frequency sub-band in wavelet decomposition. According to Weber-Fechner law, the human visual system is sensitive to the change in middle intensity of luminance region and insensitive in very low or very high intensities region. The adaptive visible watermark is designed depend on WeberFachner law and characteristics of wavelet domain. The formula

$$
C_{i j}^{\prime}=\alpha_{i j} C_{i j}+\beta_{i j} W_{i j}
$$

defines the watermark image is embedded into host image. $C_{i j}$ is a luminance coefficient in wavelet domain with coordinates $i$ and $j . W_{i j}$ is a watermark coefficient in wavelet domain with coordinates $i$ and $j$ correspond to luminance coefficient. $C_{i j}{ }^{\prime}$ represents coefficients of watermarked image. $\alpha_{i j}$ is the scaling factor for the host image. $\beta_{i j}$ is the scaling factor for the watermark image.

In low frequency sub-band, we describe the formula

$$
\begin{aligned}
& \alpha_{i j, L L}=\frac{\left|C_{i j}{ }^{\prime}-C_{i j}\right|}{C_{i j}} \geq k-\frac{\Delta I}{I} \\
& \beta_{i j, L L}=1-\alpha_{i j, L L}
\end{aligned}
$$

that defines the just noticeable difference condition of visible watermarking. $\alpha_{i j, L L}$ and $\beta_{i j, L L}$ represents the scaling factor for the host image and visible watermark in low frequency wavelet sub-band respectively. $k$ denotes visible strength factor that effect the whether observer can notice the watermark clearly. The range of $k$ is often tuned by watermark designer. The suitable range of $k$ is 2 to 5 in the literature. When visible watermark is designed for printed image, $k$ often gets a larger value ( $k$ is $4-5$ ) to appear an obvious visible watermarking. Otherwise, $k$ gets a smaller value ( $k$ is 2 to 4) to appear an indistinct visible watermark.

In the high frequency sub-bands, the wavelet coefficients describe the high frequency characteristics such as edges and textures. High amplitude corresponds to sharp variation and low amplitude represents smooth regions. In [5], a context model is proposed to estimate the distribution of wavelet coefficients. Chang [12] models each wavelet coefficient as a random variable with a Laplacian distribution of zero mean.

$$
\operatorname{Laplacian}\left(x, \sigma_{x}\right)=\frac{1}{\sqrt{2 \sigma_{x}}} \exp \left(\frac{-\sqrt{2}|x|}{\sigma_{x}}\right) \quad-\infty<x<\infty
$$

$\sigma_{x}$ is standard deviation that estimated by a method called context modelling as in [5]. Generally, $\sigma_{x}$ is larger in the edge and texture regions, and smaller in the smooth regions.

Edges and textures in high frequency sub-band (frequency orientation=LH, HL, HH) are very sensitivity. Slight change often alters the features of host image. The standard deviation $\sigma_{x}$ and scaling factor $\alpha i j, r, L, H L, H H$ are direct proportion. Therefore, the scaling factor in high frequency sub-band (with level $r=1,2,3 \ldots \ldots$ ) $\alpha_{i j, r . L H, H L . H H}$ is smaller in sensitive region. Oppositely, the scaling factor $\alpha_{i, ., L H, H L, H H}$ is larger in insensitive region such as smooth region. $r_{\max }$ is maximal level of wavelet domain. $d$ denotes the decay factor that gives smaller value in level 1 and larger value in higher level.

$$
\begin{aligned}
& \alpha_{i j, r, L H, H L, H H}=d^{\left(r_{\max }-r\right)} \cdot \sigma_{x} \\
& \beta_{i j, r, L H, H L, H H}=1-\alpha_{i j, r, L H, H L, H H}
\end{aligned}
$$

Scaling factors of luminance are calculated to estimating the effect of background luminance afterward. Spatial characteristics are analyzed to characterize the activity level of pixels. The watermark image is embedding by modifying the coefficients of the host image according to scaling factor. Finally, inverse discrete wavelet transform (IDWT) is performed to obtain the visible watermarked image

\section{HARDWARE-EFFICIENT ARCHITECTURE DESIGN}

The overall architecture for the proposed DWT domain visible watermarking is shown in Fig. 3. Host image and watermark is transferred by discrete wavelet transform (DWT) into three-level multi-resolution structures. The host image signal divides into two sequences that one is sequence A (Seq. A) and the other is sequence B (Seq. B). Sequence A is composed of $\mathrm{LL}_{3}, \mathrm{LH}_{3}, \mathrm{HL}_{3}, \mathrm{HH}_{3}, \mathrm{LH}_{2}, \mathrm{HL}_{2}, \mathrm{HH}_{2}$, and $\mathrm{LH}_{1}$ sub-band data. Sequence $\mathrm{B}$ is made up $\mathrm{HL}_{1}$ and $\mathrm{HH}_{1}$ sub-band data. Seq. A and Seq. B have the same patterns length. Two-path parallel processing architecture can reduce the half processing time. Then, de-multiplexers send the signal into difference processing element depend on different sub-bands and decomposition levels. TABLE I 


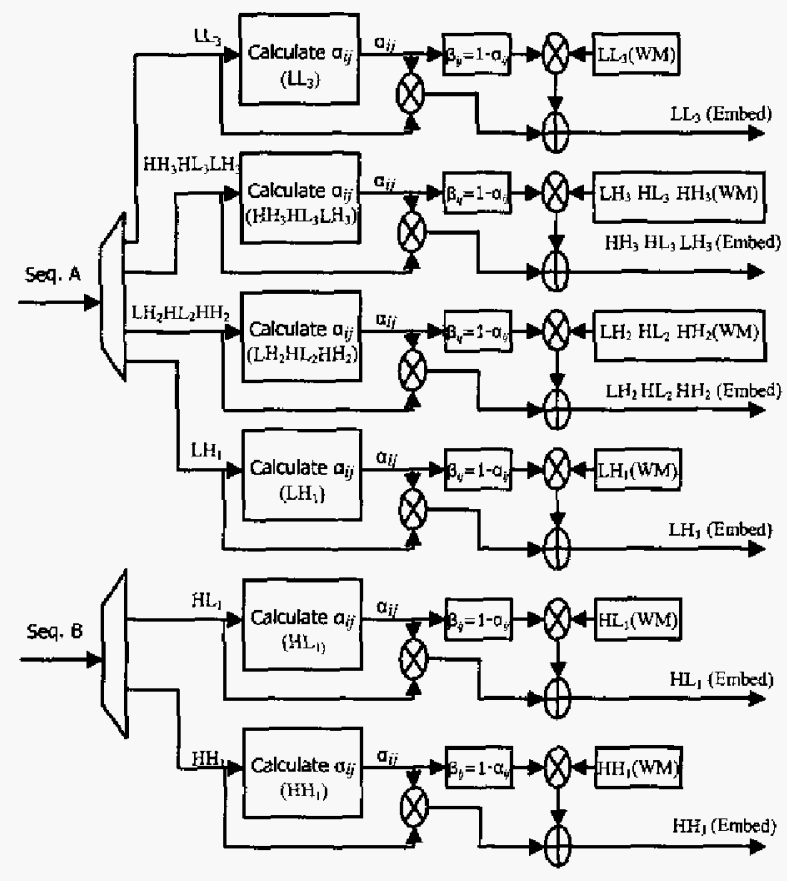

Fig. 3 Wavelet-based visible watermarking architecture.

describes the function of processing element that calculates $\alpha_{i j}$ value. According to human visual system, the range of $\alpha_{i j}$ is 0.92 to 0.96 ( $k \Delta I / I, k=2 \sim 4$ ). In order to reduce hardware complexity, we adopt eight kinds of $\alpha_{i j, L}$ value and four kinds of $\alpha_{i j, r, L H, H L, H H}$ value to instead of unlimited $a_{i j}$ value. The intensity of wavelet coefficient also separates into eight regions in $\mathrm{LL}_{3}$ sub-band and four regions in other high frequency sub-bands. The approximate $\alpha_{i j}$ and $\beta_{i j}$ instead of complex scaling factor calculation and hardware cost. The TABLE I presents the approximate rule of $\alpha_{i j}$ and $\beta_{i j}$ calculation.

Observing $\alpha_{i j}$ and $\beta_{i j}$, we can use 1-1/16-1/128 (the approximate value is 0.9296875 ) to replace 0.93 (TABLE I). After reducing, the shift registers and adders can replace all complex multiplication operation. This architecture does not need any multiplier.

Besides, we adopt the resource sharing technique to use the shift registers jointly. We just use five registers $(1 / 8,1 / 16,1 / 32,1 / 64,1 / 128)$ to calculate $\alpha_{i j}, \beta_{i j}$. At the same time, $\alpha_{i j}$ and $\beta_{i j}$ has reduplicate operation. For example, $\alpha_{i j}=1-(1 / 16+1 / 128), \beta_{i j}=(1 / 16+1 / 128)$. We can reduce the operation (1/16+1/128) adopting hardware sharing. Besides, each $\alpha_{i j}$ has the same term (1-1/16). We can use operation element jointly.

Via a series of numerical reduction, visible watermarking operation is also reduced.
TABLE I

Scaling Factor Calculation

\begin{tabular}{|c|c|c|c|c|c|c|}
\hline \multicolumn{2}{|c|}{ Discriminate } & $\begin{array}{l}a_{\mathrm{ij}, \mathrm{Ll}} \\
\text { (Real) }\end{array}$ & \multicolumn{2}{|c|}{$\begin{array}{c}\alpha_{\text {ijLe }} \\
(\text { Approx.) }\end{array}$} & $\begin{array}{l}\beta_{11 \mathrm{NL}} \\
\text { (Real) }\end{array}$ & $\begin{array}{c}\beta_{\text {k.LL }} \\
\text { (Approx) }\end{array}$ \\
\hline \multicolumn{2}{|l|}{$C_{i j}<100$} & 0.92 & \multicolumn{2}{|c|}{$1-1 / 16-1 / 64$} & & $1 / 16+1 / 64$ \\
\hline \multicolumn{2}{|l|}{$100 \leqq C_{i j}<872$} & 0.93 & \multicolumn{2}{|c|}{$5-1 / 16-1 / 128$} & 0.07 & $1 / 16+1 / 128$ \\
\hline \multicolumn{2}{|c|}{$872 \leqq C_{i}<1095$} & 0.9375 & \multicolumn{2}{|c|}{$1-1 / 16$} & 0.0625 & $1 / 16$ \\
\hline \multicolumn{2}{|c|}{$1095 \leqq C_{i j}<1233$} & 0,93 & \multicolumn{2}{|c|}{$1-1 / 16-1 / 128$} & 0,07 & $1 / 16+\mathrm{l} / 128$ \\
\hline \multicolumn{2}{|c|}{$1233 \subseteq \mathrm{C}_{\mathrm{ij}}$} & 0.92 & \multicolumn{2}{|c|}{$1-1 / 16-1 / 64$} & 0.08 & $1 / 56+1 / 64$ \\
\hline $\begin{array}{c}\text { Discriminate } \\
(d=0.96)\end{array}$ & 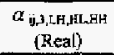 & \multicolumn{2}{|c|}{$\begin{array}{l}\alpha_{\text {ij,3Lн,HL,HH }} \\
\text { (Approx.) }\end{array}$} & \multicolumn{2}{|c|}{ 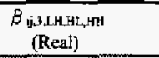 } & $\begin{array}{l}\text { LH,HLFH } \\
\text { aprox.) }\end{array}$ \\
\hline$C_{i j}<-14$ & 0.92 & \multicolumn{2}{|c|}{$1-1 / 16-1 / 64$} & \multicolumn{2}{|c|}{0.08} & $5+1 / 64$ \\
\hline$-14 \leqq C_{i j}<1$ & 0.93 & \multicolumn{2}{|c|}{$1-1 / 16-1 / 128$} & \multicolumn{2}{|c|}{0.07} & $6+1 / 128$ \\
\hline $1 \leqq C_{j j}<17$ & 0,9375 & \multicolumn{2}{|c|}{$1-1 / 16$} & \multicolumn{2}{|c|}{0.0625} & \\
\hline $17 \leq \mathrm{C}_{\mathrm{ij}}$ & 0.945 & \multicolumn{2}{|c|}{$1-1 / 26+1 / 128$} & \multicolumn{2}{|c|}{0.055} & 6-1/128 \\
\hline $\begin{array}{l}\text { Discriminate } \\
(\mathrm{d}=0.96)\end{array}$ & $\begin{array}{l}a_{\mathrm{ij}, 2, \mathrm{LH}, \mathrm{HL}, \mathrm{HH}} \\
\text { (Reall; }\end{array}$ & \multicolumn{2}{|c|}{ 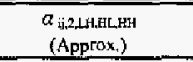 } & \multicolumn{2}{|c|}{$\begin{array}{l}\beta_{i \mathrm{ij}, 2 \mathrm{LH}, \mathrm{LL}, \mathrm{HH}} \\
\text { (Real) }\end{array}$} & $\begin{array}{l}\text { LLHL.HA } \\
\text { gprox) }\end{array}$ \\
\hline$C_{i j}<-6$ & 0.8832 & \multicolumn{2}{|c|}{$1-1 / 16-1 / 32-1 / 64-1 / 128$} & \multicolumn{2}{|c|}{0.1168} & $2+1 / 64+1 / 128$ \\
\hline$-6 \leq c_{i j}<1$ & 0.8928 & \multicolumn{2}{|c|}{$1-1 / 16-1 / 32-1 / 64$} & \multicolumn{2}{|c|}{0.1072} & $2+1 / 64$ \\
\hline $1 \leqq c_{i j}<7$ & 0.9024 & \multicolumn{2}{|c|}{$1-1 / 16-1 / 32$} & \multicolumn{2}{|c|}{0.0976} & \\
\hline $7 \leq C_{i j}$ & 0,912 & $1,1 / 16-1$ & & 0.088 & & $4+1 / 128$ \\
\hline $\begin{array}{c}\begin{array}{c}\text { Discriminale } \\
(\mathrm{d}=0,96)\end{array} \\
\end{array}$ & $\begin{array}{c}\alpha_{\mathrm{ij}, \mathrm{i}, \mathrm{LH}, \mathrm{HL}, \mathrm{HH}} \\
\text { (Real) }\end{array}$ & $\begin{array}{r}a_{i, i, 1} \\
\quad\left(A_{F}\right. \\
\end{array}$ & & 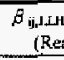 & & $\begin{array}{l}\text { LH,HL,Hн } \\
\text { oprox, }\end{array}$ \\
\hline$c_{i j}<-3$ & 0.8464 & $1-1 / 16-1$ & $4-1 / 128$ & 0.153 & & $2+1 / G A+1 / 128$ \\
\hline$-3 \leqq C_{i j}<0$ & 0.8556 & $1-1 / 16-1$ & & 0.144 & & $2+1 / 64$ \\
\hline $0 \leqq \mathrm{C}_{\mathrm{j}}<3$ & 0.8648 & $1-1 / 16-1$ & & 0.135 & & \\
\hline $3 \leqq c_{i j}$ & 0.874 & $1-1 / 16-1$ & & 0.126 & & $4+1 / 128$ \\
\hline
\end{tabular}

\section{EXPERIMENTAL RESULTS}

In order to prove the ability of the wavelet-based adaptive visible watermarking architecture, a series of experiments were conducted. The watermark is shown in Fig. 4. The proposed architecture has been tested on various images. The experimental results are shown in Fig. 5 with "Lena," "Tiffany," "Baboon," and "skylight (Chinese free verse)" images of $256 \times 256 \times 8$ bits. The former contains mainly smooth regions, edge, and texture, which represent low frequency and high frequency. The visual inspection of the watermarked images proves that watermarking is able to preserve the quality of the image while explicitly proving the ownership. We used signal to noise ratio (SNR) as suggested by [6] to quantify the quality of the watermarked images. We calculated the SNR using the original and the watermarked image. The calculation results show that the SNR for various watermarked images is in the range of $22 \mathrm{~dB}$ to $26 \mathrm{~dB}$.

In order to prove the architecture is hardware efficient, we give a comparison results as listed in TABLE II. The direct form DWT-based visible watermarking method [8] needs 24 additions, 12 multiplications, 3 divisions, 1 radical and 2 shift registers operation. However, the proposed method just needs 30 additions and 5 shift registers operation without any multiplication, division and radical operation. The approximate technique, numerical reduction and resource sharing technique reduce a great deal operation and hardware complexity. Therefore, It reveals that our proposed 


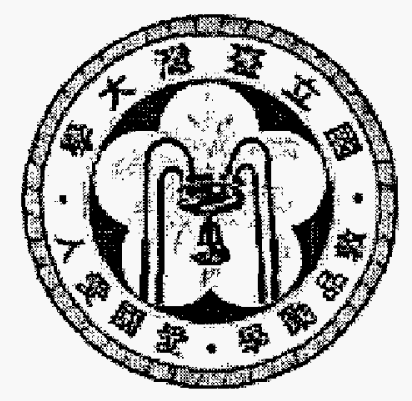

Fig. 4 Visible watermarking (National Taiwan University)

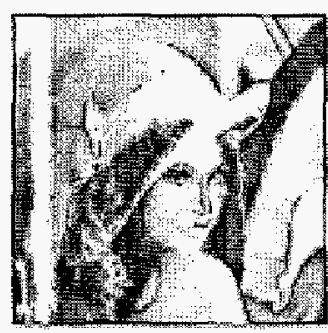

(a)

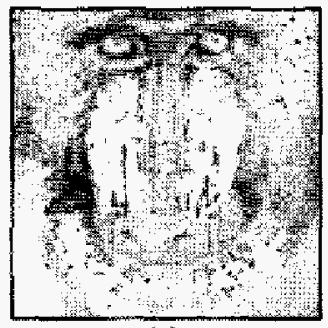

(c)

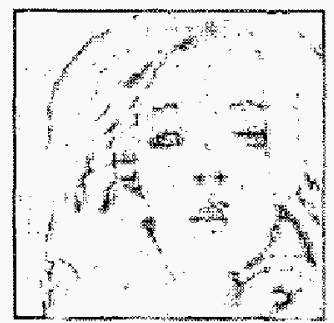

(b)
Fig. 5 Experimental results (a) Lena image, (b) Tiffany image, (c) baboon image, (d) skylight (Chinese free verse).

architectures have characteristics of hardware-efficient and fewer computing operations in TABLE II.

This scheme reduces the hardware complexity and holds high image quality. The experimental results have proven the proposed architecture is indeed hardware-effective.

\section{Conclusion}

The hardware-efficient architecture design of waveletbased adaptive visible watermarking is presented in this paper. We develop the efficient hardware architecture of wavelet-based adaptive visible watermarking that adopts approximate technique, numerical reduction and resource sharing technique to reduce hardware complexity. Luminance characteristics are calculated to estimating the effect of background luminance afterward. Local spatial
TABLE II

Comparison Results

\begin{tabular}{lcc}
\hline \multicolumn{1}{c}{ Parameters } & Hu's Method [8] & Proposed Method \\
\hline Addition & 24 & 30 \\
Multiplication & 12 & 0 \\
Division & 3 & 0 \\
Radical & 1 & 0 \\
Shift Register & 2 & 5 \\
\hline
\end{tabular}

characteristics are analyzed to characterize the activity level of pixels. The watermark image is embedding by modifying the coefficients of the host image according to scaling factor. According to experimental results, our method reduces the hardware complexity and holds high image quality.

\section{ACKNOWLEDGMENT}

The authors gratefully acknowledge National Applied Research Laboratories, National Chip Implementation Center (CIC) for technical support and supplying the EDA tools used in the hardware design.

\section{REFERENCES}

[1] I. J. Cox, M. L. Miller, and J. A. Bloom, Digital Watermarking, CA: Academic Press, San Diego, 2002.

[2] C. H. Huang, and J. L. Wu, "Attacking visible watermarking schemes," IEEE Transactions on Multimedia, vol. 6, no. 1, pp: 16-30, Feb. 2004.

[3] Y. C. Fan, W. L. Mao, and H. W. Tsao, "An Artificial Neural Network-Based Scheme for Fragile Watemarking", IEEE International Conference on Consumer Electronics, ICCE 2003, pp. 210 - 211 , June 17-19, 2003, Los Angeles, California, U.S.A.

[4] Y. C. Fan, and H. W. Tsao, "A Data Backup Embedding Scheme for Fragile Watermarking", IEEE International Conference on Informatics Cybernetics and Systems, ICICS 2003, pp: 1103-1108, Dec. 14-16, 2003.

[5] Y. C. Fan, and H. W. Tsao, "A Dual Pyramid Watermarking for JPEG-2000," IEEE The First International Workshop on Information Networking and Applications. INA 2005, March 28-30, 2005.

[6] S. P. Mohanty, N. Ranganathan, and R. K. Namballa, "VLSI implementation of visible watermarking for secure digital still camera design," I7th International Conference on VLSI Design, pp: 1063 1068, Jan. 2004.

[7] S. P. Mohanty, N. Ranganathan, and K. Balakrishnan, "Design of a low power image watermarking encoder using dual voltage and frequency," 18th international Conference on VLSI Design, pp: $153-158$, Jan. 2005.

[8] $\mathrm{Y}$. $\mathrm{Hu}$, and $\mathrm{S}$. Kwong, "Wavelet domain adaptive visible watermarking," Electronics Letters, vol. 37, no. 20, pp: 1219 - 1220, Sept. 2001

[9] W. F. Schreiber, Fundamentals of Electronic Imaging Systems, Springer-Verlag, Berlin, 1991.

[10] W. K. Pratt, Digital Image Processing, Wiley-Interscience, 2001.

[11] S. Hecht, "The visual discrimination of intensity and the WeberFechner law," Journal of General Physiology, 7, 1924, 241.

[12] S. G. Chang, B. Yu, and M. Vetlerli, "Spatially adaptive wavelet thresholding with context modeling for image denoising," [EFE Transactions on Image Processing, vol. 9, no. 9, pp: 1522 - 1531, Sept. 2000. 


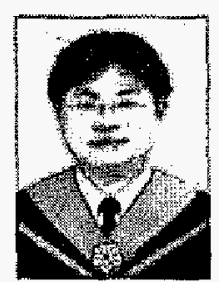

Yu-Cheng Fan ( $\mathrm{S}^{\prime} 00$ ) was bom in Hsinchu, Taiwan, R.O.C., on June 6,1975 . He received the B.S. degree in electrical engineering from National Cheng Kung University, Tainan, R.O.C., in 1997, and M.S. degree in electrical engineering also from National Cheng Kung University in 1999. He is currently a graduate student in the Department of Electrical Engineering of National Taiwan University, Taipei, Taiwan, R.O.C., pursing his doctor's degree. His research interests are consumer electronics, digital watermarking, image and video coding system. In 1999, he received Long-Term (Acer) Paper Awards. He was an elected Chairnan of the IEEE NTU Student Branch in 2003. In 2005, he has received IEEE Award for outstanding leadership and service to the IFEE NTU Student Branch.

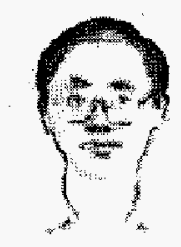

instrumentation.
Hen-Wai Tsao ( $\left.\mathrm{M}^{\prime} 90\right)$ was born in Taipei, Taiwan, R.O.C., in 1953. He recerved the B.S., the M.S. and the $\mathrm{Ph} . \mathrm{D}$ degres in electrical engineering from National Taiwan University in 1975, 1978 and 1990, respectively. Since 1978 , he has been with the Department of Electrical Engineering, National Taiwan University, Taipei, Taiwan, R.O.C., where he is currently a professor. His main research interests are optical fiber communication system, communication electronics, and electronic

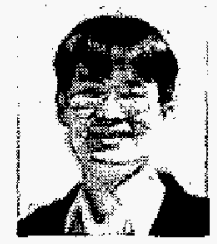

Lan-Da Van (S'98-AM'01-M'02) was born in Miaoli , Taiwan , R.O.C., on Oct. 09, 1972, He received the B.S. (Honors) and the M.S. degree from Tatung institute of Technology, Taipei, Taiwan , R.O.C. in 1995 and 1997, respectively, and the $\mathrm{Ph}$. D. degree from National Taiwan University (NTU), Taipej, Taiwan, R.O.C. in 2001, all in electrical engineering.

From 1997 to 2001, he was a research assistant at National Taiwan University. From 2001, He is a researcher at Chip Implementation Center (CIC), National Applied Research Laboratories, Hsinchu, Taiwan, R.O.C. His currently research interests are in VLSI architectures, algorithms, and chips for digital signal processing (DSP) and baseband communication systerns. This includes the design of high-performance /low-power/areaaware DSP processors, adaptive digital filters, orthogonal transforms, computer arithmetic, platform-based SoC designs, and multidimensional signal processing systems. He has published more than 15 IEEE joumal and conference papers in these areas.

Dr. Van was a recipient of the Chunghwa Picture Tube (CPT) and Motorola fellowships in 1996 and 1997, respectively. He won National Taiwan University scholarships in 1997 and 1999. He was an elected vicechairman and chairman of IEEE NTU Student Branch in 1999 and 2000, respectively. In 2002, he has received IEEE Award for outstanding leadership and service to the IEEE NTU Student Branch. In 2003 and 2004, he was listed in Marquis Who's Who in science and engineering. In 2005, he is a recipient of the Best Poster Award at iNEER Conference for Engineering Education and Research (ICEER). He was a referee of IEEE ISCAS and SCI. Presently, he serves as a reviewer for IEEE Transaction on Circuits and Systems I: Fundamental Theory and Applications, IEEE Transaction on Circuits and Systens II: Analog and Digital Signal Processing, IEEE Transaction on VLSI Systems, IEEE Transaction on Multimedia, IEEE Stgnal Processing Letters, Elsevier Microelectronics Joumal, Elsevier Integration, The VLSI Joumal and EURASIP Journal on Applied Signal Processing. He has reviewed over 15 joumal/transaction papers in these periodicals.

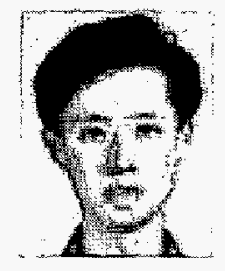

Chun-Ming Huang is a manager and researcher at Chip Implementation Center (CIC), National Applied Research Laboratories, Hsinchu, Taiwan, R.O.C. His currently research interests are in VLSI design and testing, platform-based SOC design, and multimedia communication. In 2005, he is a recipient of the Best Poster Award at iNEER Conference for Engineering Education and Research (ICEER). 